\title{
Treatment with bone marrow mesenchymal stem cells combined with plumbagin alleviates spinal cord injury by affecting oxidative stress, inflammation, apoptotis and the activation of the Nrf 2 pathway
}

\author{
WENCHENG YANG, YAN YANG, JIAN-YI YANG, MING LIANG and JIANGTAO SONG \\ The Second Department of Orthopedics, Zhengzhou Central Hospital \\ Affiliated to Zhengzhou University, Zhengzhou, Henan 450007, P.R. China
}

Received May 12, 2015; Accepted February 12, 2016

DOI: $10.3892 /$ ijmm.2016.2498

\begin{abstract}
The aim of the present study was to investigate the protective effect exerted by bone marrow mesenchymal stem cells (BMSCs) in combination with plumbagin on spinal cord injury (SCI) and explore the mechanism behind this protective effect. Firstly, BMSCs were extracted from male Sprague-Dawley rats, cultured in vitro, and identified by hematoxylin. Sprague-Dawley rats were then randomly divided into a control group, SCI model group, BMSC-treated group, a plumbagin-treated group, and a BMSC and plumbagin-treated group. After treatment with BMSCs combined with plumbagin, a Basso, Beattie and Bresnahan (BBB) test was carried out and the spinal cord water content was examined in order to analyze the effect of BMSCs combined with plumbagin on SCI. The myeloperoxidase (MPO), superoxide dismutase (SOD), malondialdehyde (MDA), nuclear factor- $\kappa \mathrm{B}(\mathrm{NF}-\kappa \mathrm{B})$ p65 unit, tumor necrosis factor- $\alpha(\mathrm{TNF}-\alpha)$ levels were also detected. Moreover, nuclear factor erythroid 2-related factor 2 (Nrf2), phosphoinositide 3-kinase (PI3K), phosphorylated (p-)Akt, p-p38 mitogen-activated protein kinase (MAPK), and p-extracellular-signal-regulated kinase (ERK) protein expression levels were measured using western blot analysis. Treatment with BMSCs combined with plumbagin significantly improved locomotor recovery and reduced the spinal cord water content after SCI. The increased MPO, MDA, NF- $\kappa$ B p65 and TNF- $\alpha$ levels were significantly suppressed and the decreased SOD was significantly increased in SCI rats. The suppression of Nrf2, p-Akt and p-ERK, as well as the promotion of p-p38 MAPK, were reversed by treatment with BMSCs combined with plumbagin. These effects suggest that treatment with
\end{abstract}

Correspondence to: Dr Wencheng Yang, The Second Department of Orthopedics, Zhengzhou Central Hospital Affiliated to Zhengzhou University, Tongbai Road 195, Zhengzhou, Henan 450007, P.R. China E-mail: qqwenchengyang@126.com

Key words: bone marrow mesenchymal stem cells, plumbagin, spinal cord injury, oxidative stress, inflammation, anti-apoptotic
BMSCs combined with plumbagin alleviates SCI through its effects on oxidative stress, inflammation, apoptotis and activation of the Nrf2 pathway.

\section{Introduction}

For patients who have suffered spinal cord injury (SCI), the disintegration and necrosis of a large number of neuronal cells in tissues is one of the major causes of reduced limb movement and sensory dysfunctions in the area below the injury (1). Thus, promoting the regeneration of nerve cells and recovering the structure and function of the spinal cord are important and difficult issues with which neuroscientists are concerned (2).

Bone marrow mesenchymal stem cells (BMSCs) are non-hematopoietic stem cells in the bone marrow which are derived from the mesoderm; they can self-proliferate and are capable of multi-directional differentiation (3). A BMSC is a type of adult pluripotent stem cell, isolated and cultured from the bone marrow, which can be found in different species such as chickens, rats, rabbits, dogs and other animals as well as humans (4). Its potential in multi-directional differentiation has received much attention in studies on Alzheimer's disease, strokes, myocardial infarction, peripheral nerve injury, bone defects and nonunion (5). It has preciously been reported that BMSCs have the ability to differentiate into neuroectodermal cells. In a previous study, the BMSCs were injected into the hippocampus of mice and the differentiation into astrocytes was observed; under specific induction conditions, they can also be induced to differentiate into neural cells which are capable of expressing neuron-specific marker proteins (6).

Plumbagin is from the Plumbaginaceae family, genus Plumbago, which is mainly distributed in the southwestern provinces of China, and is usually used for treating sores, swelling, clearing the meridians, and treating snakebites, rheumatism, mastitis and chronic bronchitis (7). Previous pharmacological studies have demonstrated that plumbagin is one of the most important active antitumor components of Plumbago, which exerts anti-bacterial, anti-inflammatory, anti-cancerous and other beneficial effects $(8,9)$. In the present study, we aimed to explore the protective effect of a treatment which combined 
BMSCs with plumbagin on SCI and also explore the potential mechanism of the protective effects.

\section{Materials and methods}

Reagents. $\alpha$-modified Eagle's medium (MEM) and Dulbecco's modified Eagle's medium (DMEM) were provided by Thermo Scientific (Waltham, MA, USA). Fetal bovine serum (FBS) was provided by Gibco (Grand Island, NY, USA). Plumbagin was purchased from Sigma-Aldrich (Hamburg, Germany). Myeloperoxidase (MPO), superoxide dismutase (SOD), malondialdehyde (MDA), nuclear factor- $\mathrm{KB}$ (NF- $\mathrm{kB}$ ) p65, tumor necrosis factor- $\alpha$ (TNF- $\alpha$ ) kits and hematoxylin were all purchased from Invitrogen (Carlsbad, CA, USA).

Animals. Male Sprague-Dawley rats (SD rats) weighing $220 \pm 30 \mathrm{~g}$ were used for the present study. The SD rats were maintained in animal quarters with humidity ranging from $60-70 \%$, a temperature of $23 \pm 1^{\circ} \mathrm{C}$ and a 12-h light/dark cycle. Food and water were made available ad libitum. All experiments were carried out in accordance with the criteria outlined in the Guide for the Care and Use of Laboratory Animals prepared by the National Academy of Sciences and were approved by the Ethics Committee of Zhengzhou University (Zhengzhou, China).

Cell culture and identification of BMSCs. The SD rats were sedated using chloral hydrate, and their bilateral femurs and tibias were removed under sterile conditions and separated. Subsequently, bone marrow tissues of the bilateral femur and tibia were separated, and cell samples were collected from these bone marrow tissues and seeded into a $25-\mathrm{cm}^{2}$ plastic bottle to separate and obtain BMSCs using the adherence method. BMSCs were cultivated in $5 \mathrm{ml} \alpha$-MEM (Thermo Scientific) and $10 \%$ FBS and cultivated in the plastic bottle for incubation. After cultivation for 24-48 h, the non-adherent cells were removed. When adherent cells reached $70-80 \%$ confluence, adherent cells were then incubated with $0.25 \%$ trypsin to remove them.

After paraformaldehyde (5\%) was precooled, it was used to fix BMSCs for 10-15 min. The fixed BMSCs were cultured with hematoxylin (Invitrogen) for 3-10 min. Stained BMSCs were then washed with tap water and distilled water for 5-10 min. Stained BMSCs were dehydrated with 95\% ethyl alcohol for 1-2 min, and xylene was used to clear the stained cells for 5-10 min. BMSCs were observed using a microscope (CFI60; Nikon, Tokyo, Japan).

Induction of SCI. A rat model of SCI was induced as described previously (10). Briefly, SD rats were intraperitoneally injected with $80 \mathrm{mg} / \mathrm{kg}$ ketamine and $10 \mathrm{mg} / \mathrm{kg}$ xylazine and then underwent a laminectomy during which the T8 and T9 vertebral peduncles were removed. After treatment was completed, the SD rats were sacrificed using a lethal injection of pentobarbital, and the spinal tissue was removed while on dry ice. The tissue from the injury site of the spinal cord segments (between T8 and T9) was microdissected and frozen on dry ice. The spinal tissue samples were homogenized with Tris-HCl buffer (50 mM, pH 7.4) on ice for future use.

Treatment schedule. Firstly, 10 normal SD rats were defined as the control group and injected with normal saline. Subsequently,
40 rats for the SCI model were randomly divided into 4 groups as follows: $\mathrm{SCI}$ model group (SCI, $\mathrm{n}=10$ ), BMSC-treated group (BMSCs, $n=10$ ), plumbagin-treated group (plumbagin, $n=10$ ) and the BMSC and plumbagin-treated group (BP, $n=10)$. In the SCI model group, rats were injected with saline and SCI was induced. In the BMSC-treated group, rats with SCI were treated with BMSCs $\left(3 \times 10^{5}\right.$ cells) by intraspinal injection using a Hamilton syringe, as previously described (11) for 5 consecutive days. In the plumbagin group, rats with SCI were treated with $20 \mathrm{mg} / \mathrm{kg}$ plumbagin, as previously described, (12) for 5 consecutive days. In the group treated with BMSCs and plumbagin, rats with SCI were treated with BMSCs $\left(3 \times 10^{5}\right.$ cells $)$ by intraspinal injection using a Hamilton syringe and also $20 \mathrm{mg} / \mathrm{kg}$ plumbagin for 5 consecutive days.

Locomotor recovery after SCI. Locomotor recovery was assessed as previously described (13). Locomotor behavior was evaluated beginning 1 day before injury and ending 5 days after. Scoring categories and attributes were identified, operationally defined and ranked on the observed sequence of locomotor recovery patterns using the Basso, Beattie and Bresnahan (BBB) locomotor rating scale. In the BBB scale, 0 is defined as "no observable movement of the hindlimbs', 21 is defined as 'consistent plantar stepping and coordinated gait, consistent movement of the toes; paw position is predominantly parallel to the body during the whole support stage; consistent trunk stability; consistent tail elevation'. The BBB rating was carried out 1 day before SCI was induced (0 day), 1 day after SCI was induced (1 day), and again 5 days later (5 day).

Measurement of spinal cord water content after SCI. After treatment with BP for 5 consecutive days, spinal cord water content was measured as previously described (14). Briefly, thye SD rats were sacrificed using a lethal injection of pentobarbital and the spinal tissue was removed while on dry ice, and dried at $80^{\circ} \mathrm{C}$ for 24-48 $\mathrm{h}$ before the dry weight was measured. Spinal cord water content was calculated as follows: water content $=[($ wet weight - dry weight)/wet weight] x100.

$M P O$ activity. After treatment with BP for 5 consecutive days, and after the rats were sacrificed and the spinal cord tissue removed, the injured spinal cord segment was mechanically homogenized in ice-cold tris-buffered saline containing $40 \mathrm{mM}$ Tris- $\mathrm{HCl}$ (pH 7.5), $2 \%$ SDS, $2 \mathrm{mg} / \mathrm{ml}$ aprotinin, $2 \mathrm{mg} / \mathrm{ml}$ antipain, $2 \mathrm{mg} / \mathrm{ml}$ chymostatin, $2 \mathrm{mg} / \mathrm{ml}$ bestatin, $2 \mathrm{mg} / \mathrm{ml}$ pepstatin-A, $2 \mathrm{mg} / \mathrm{ml}$ leupeptin, $1 \mathrm{mM}$ phenylmethylsulfonyl fluoride, $1 \mathrm{mM}$ dithiothreitol and $1 \mathrm{mM}$ EDTA. The homogenates were centrifuged at $12,000 \mathrm{x}$ g for $10 \mathrm{~min}$. Liquid supernatant was defined as the quantity of enzyme degrading and was expressed in $\mathrm{U} / \mathrm{g}$ of wet tissue following the manufacturer's protocol (Invitrogen).

Oxidative stress and inflammation. After treatment with BP for 5 consecutive days, and after the rats were sacrificed and the spinal cord tissue removed, the spinal tissue samples were centrifuged at $12,000 \mathrm{x} \mathrm{g}$ for $10 \mathrm{~min}$. Liquid supernatant was used to assess SOD, MDA, NF- $\kappa B$ p 65 and TNF- $\alpha$ activity following the manufacturer's instructions (Beyotime, Nanjing, China).

Western blot analysis. After treatment with BP for 5 consecutive days, and after the rats were sacrificed and the spinal cord tissue 

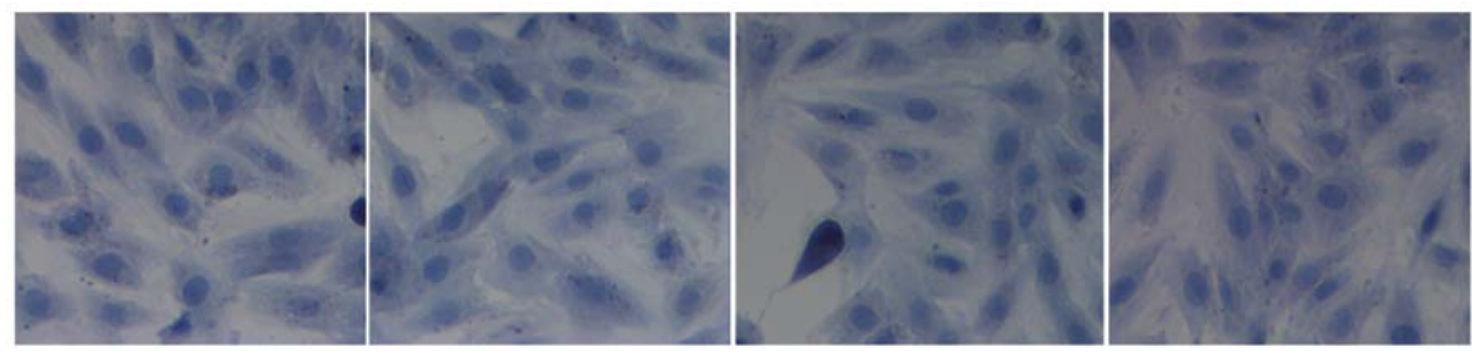

Figure 1. Bone marrow mesenchymal stem cell (BMSC) culture and detection. BMSCs were detected and observed using hematoxylin staining and microscopic analysis, and cells were successfully separated and cultivated. Magnification, x200.

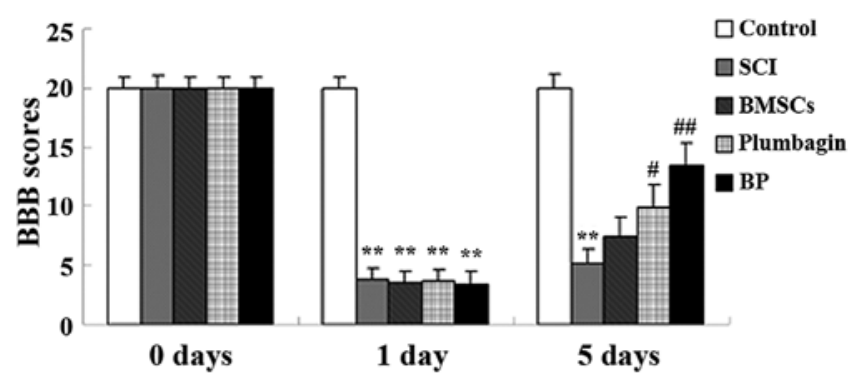

Figure 2. Treatment with bone marrow mesenchymal stem cells (BMSCs) and plumbagin improves locomotor recovery after spinal cord injury (SCI). In the BP group, rats with SCI were treated with BMSCs ( $3 \times 10^{5}$ cells) administered by intraspinal injection using a Hamilton syringe and $20 \mathrm{mg} / \mathrm{kg}$ plumbagin for 5 consecutive days. The data are expressed as the means \pm SD and were analyzed using one-way ANOVA followed by Tukey's multiple comparison test. ${ }^{* *} \mathrm{p}<0.01$ compared with the control group; ${ }^{*} \mathrm{p}<0.05$ compared with the SCI group; ${ }^{\#} \mathrm{p}<0.01$ compared with the SCI group. Control, control group; SCI, SCI group; BMSCs, BMSC-treated group; plumbagin, plumbagin-treated group; BP, BMSC + plumbagin-treated group.

removed, the spinal tissue homogenates were centrifuged at $12,000 \mathrm{x} g$ for $10 \mathrm{~min}$. Liquid supernatant was subsequently used to measure the protein concentration with the Coomassie (G250) binding method. Fifty micrograms of sample proteins were loaded onto $12 \%$ sodium dodecyl sulfate polyacrylamide gel electrophoresis (SDS-PAGE) and then transferred to 0.22-mm nitrocellulose membranes (Bio-Rad, Munich, Germany). The membranes were blocked with 5\% non-fat milk in Tris-buffered saline (pH 7.4) containing 0.1\% Tween-20 (TBST) for 2-3 h at room temperature. The membranes were subsequently incubated with primary antibodies overnight at $4^{\circ} \mathrm{C}$ : antinuclear factor erythroid 2-related factor 2 (Nrf2; sc-365949), anti-phosphorylated (p-)Akt (sc-293125), anti-Akt (sc-135829), p-p38 mitogen-activated protein kinase (MAPK; sc-7973), anti-p-extracellular-signal-regulated kinase (ERK; sc-365234) and anti-ERK (sc-514302) antibodies (all from Santa Cruz Biotechnology, Inc., Santa Cruz, CA, USA). The membranes were washed with TBST and incubated at $4^{\circ} \mathrm{C}$ with secondary antibody for 2-3 $\mathrm{h}$, horseradish peroxidase-linked anti-rabbit IgG (Santa Cruz Biotechnology, Inc.).

Statistical analysis. The data were calculated using SPSS 17.0 software, are expressed as the means \pm SD, and were analyzed using one-way analysis of variance (ANOVA) followed by Tukey's multiple comparison test. A p-value $<0.05$ was considered to indicate a statistically significant difference.

\section{Results}

BMSC culture and detection. As shown in Fig. 1, the cell morphology of BMSCs was observed: dark blue color, spindle shape, and serial sub-cultivation, and homogeneity and multiplicity were all observed. These features indicated that BMSCs were successfully separated and cultivated.

Treatment with BP improves locomotor recovery after SCI. In order to test the hypothesis that treatment with BP improves locomotor recovery after SCI, BBB scores were calculated. The results show that $\mathrm{SCI}$ caused a significant decrease in $\mathrm{BBB}$ scores when compared to the control group, and these were improved by BP treatment (Fig. 2). Five days after SCI induction, only the administration of BMSCs augmented BBB scores in a non-statistically significant manner. At day 5, the administration of plumbagin only significantly increased the BBB scores when compared to the SCI group. The administration of BMSCs and plumbagin (the BP group) increased the BBB scores even more significantly (compared to the SCI group) than the group treated only with plumbagin (Fig. 2).

Treatment with BP reduces spinal cord water content after $S C I$. We then determined whether treatment with BP reduces spinal cord water content after SCI. Spinal cord water content was significantly elevated in the SCI group when compared to the control group (Fig. 3). The administration of BMSCs alone suppressed the spinal cord water content in a non-statistically significant manner. Compared to the rats in the SCI group, treatment with plumbagin significantly lowered the spinal cord water content. However, in the rats in the BP group, this decrease was even more significant (compared to the SCI group) than that observed in the plumbagin-treated only group after SCI induction (Fig. 3).

Treatment with BP affects MPO activity after SCI. To clarify the effects of BP treatment on MPO activity after SCI, MPO activity was measured. Compared with the control group, MPO activity was increased after SCI (Fig. 4). BMSC treatment inhibited MPO activity in a non-statistically significant manner. The increased MPO activity was markedly decreased by treatment with plumbagin when compared to the rats with SCI. In addition, MPO activity in the BP-treated group was even lower than that in the plumbagin-treated group (Fig. 4).

Treatment with BP affects oxidative stress after SCI. In order to explore the effect of treatment with BP on oxidative stress after 


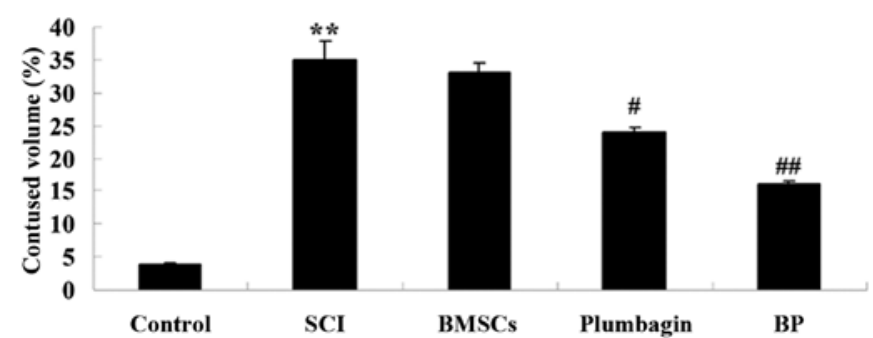

Figure 3. Treatment with bone marrow mesenchymal stem cells (BMSCs) and plumbagin reduces spinal cord water content after spinal cord injury (SCI). In the BP group, rats with SCI were treated with BMSCs $\left(3 \times 10^{5}\right.$ cells $)$ administered by intraspinal injection using a Hamilton syringe and $20 \mathrm{mg} / \mathrm{kg}$ plumbagin for 5 consecutive days. The data are expressed as the means \pm SD and were analyzed using one-way ANOVA followed by Tukey's multiple comparison test. ${ }^{* *} \mathrm{p}<0.01$ compared with the control group; ${ }^{\#} \mathrm{p}<0.05$ compared with the SCI group; ${ }^{\# \#} \mathrm{p}<0.01$ compared with the SCI group. Control, control group; SCI, SCI group; BMSCs, BMSC-treated group; plumbagin, plumbagin-treated group; BP, BMSC + plumbagin-treated group.

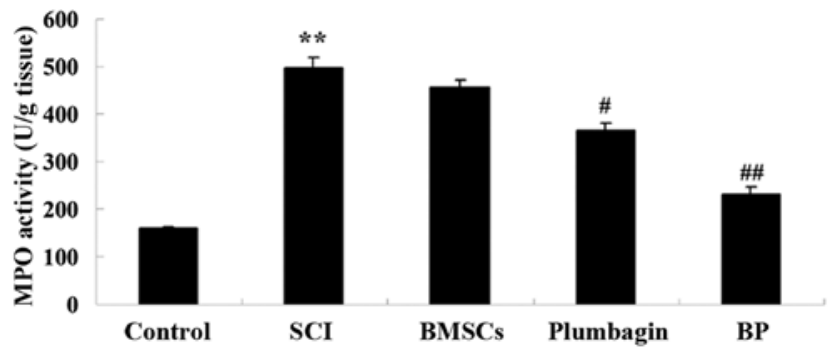

Figure 4. Treatment with bone marrow mesenchymal stem cells (BMSCs) and plumbagin affects MPO activity after spinal cord injury (SCI). In the BP group, rats with SCI were treated with BMSCs $\left(3 \times 10^{5}\right.$ cells) administered by intraspinal injection using a Hamilton syringe and $20 \mathrm{mg} / \mathrm{kg}$ plumbagin for 5 consecutive days. The data are expressed as the means \pm SD and were analyzed using one-way ANOVA followed by Tukey's multiple comparison test. ${ }^{* *} \mathrm{p}<0.01$ compared with the control group; ${ }^{\#} \mathrm{p}<0.05$ compared with the SCI group; ${ }^{\#} \mathrm{p}<0.01$ compared with the SCI group. Control, control group; SCI, SCI group; BMSCs, BMSC-treated group; plumbagin, plumbagin-treated group; BP, BMSC + plumbagin-treated group.
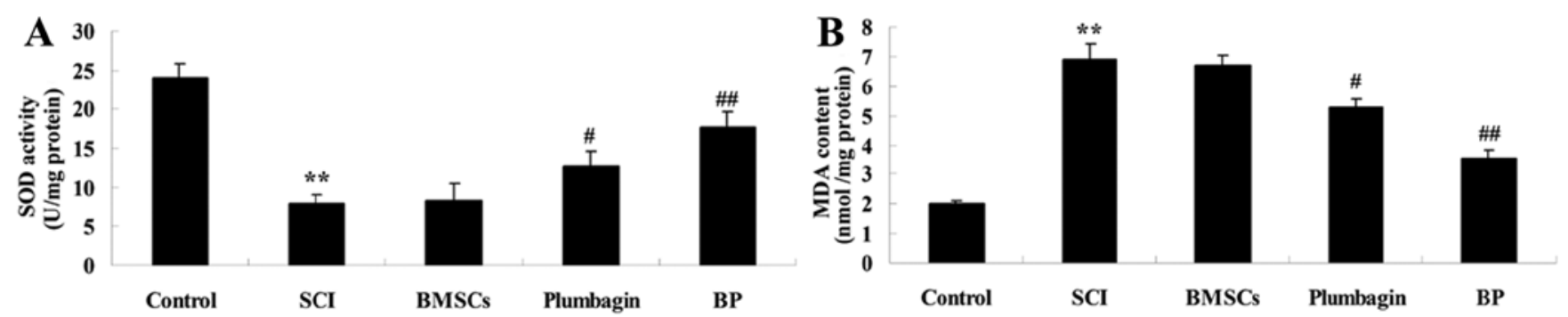

Figure 5. Treatment with bone marrow mesenchymal stem cells (BMSCs) and plumbagin affects oxidative stress after spinal cord injury (SCI). Treatment with BP affected (A) superoxide dismutase (SOD) and (B) malondialdehyde (MDA) after SCI. In the BP group, rats with SCI were treated with BMSCs (3x10 5 cells) administered by intraspinal injection using a Hamilton syringe and $20 \mathrm{mg} / \mathrm{kg}$ plumbagin for 5 consecutive days. The data are expressed as the means \pm SD and were analyzed using one-way ANOVA followed by Tukey's multiple comparison test. ${ }^{* *} \mathrm{p}<0.01$ compared with the control group; ${ }^{*} \mathrm{p}<0.05$ compared with the SCI group; ${ }^{\# \#}$ p $<0.01$ compared with the SCI group. Control, control group; SCI, SCI group; BMSCs, BMSC-treated group; plumbagin, plumbagin-treated group; BP, $\mathrm{BMSC}+$ plumbagin-treated group.

SCI, SOD and MDA activity was analyzed. SCI suppressed SOD activity and increased MDA activity compared with the control group (Fig. 5). Treatment with BMSCs slightly increased SOD activity and reduced the promotion of MDA activity (neither was statistically significant). The administration of plumbagin effectively reversed the respective decrease in SOD activity and increase in MDA content when compared to the SCI groups. Thus, treatment with BP effectively enhanced the curative effects (Fig. 5).

Treatment with BP affects inflammation after SCI. To further explore the effect of treatment with BP on inflammation, $\mathrm{NF}-\kappa \mathrm{B}$ p65 and TNF- $\alpha$ activity was examined. We demonstrated that the activity of NF- $\mathrm{BB}$ p 65 and TNF- $\alpha$ in SCI rats was markedly increased when compared to the control groups (Fig. 6). The levels of inflammatory factors in the BMSC-treated groups were very similar to those of the SCI groups. However, the upregulation of NF- $\kappa \mathrm{B}$ p 65 and $\mathrm{TNF}-\alpha$ was markedly reduced by administration of plumbagin when compared to the SCI group. Moreover, compared to plumbagin treatment, BP treatment decreased the activities of NF- $\kappa \mathrm{B}$ p65 and TNF- $\alpha$ even more considerably when compared to the SCI group (Fig. 6).
Treatment with BP affects the Nrf2 pathway after SCI. To further investigate the possible mechanism of treatment with BP, Nrf2 protein expression was appraised using western blot analysis. We noted a significant upregulation of Nrf2 pathway protein expression (Fig. 7). There was no significant difference between the SCI group and the BMSC-treated group. We also observed that Nrf2 protein expression was significantly increased by treatment with plumbagin only when compared to the SCI group. Moreover, treatment with BP increased Nrf2 protein expression even more significantly when compared to the SCI group (Fig. 7).

Treatment with BP affects the phosphoinositide 3-kinase (PI3K)/Akt pathways after SCI. To further research the possible mechanism of treatment with BP, p-Akt and Akt protein expression were evaluated using western blot analysis. The results of the western blot analysis showed that p-Akt/Akt expression in the SCI group was significantly inhibited when compared to the control group (Fig. 8). Administration of plumbagin did not significantly affect $\mathrm{p}$-Akt/Akt expression. In addition, treatment with BMSCs significantly increased p-Akt/Akt expression when compared to the SCI group. However, there was no 

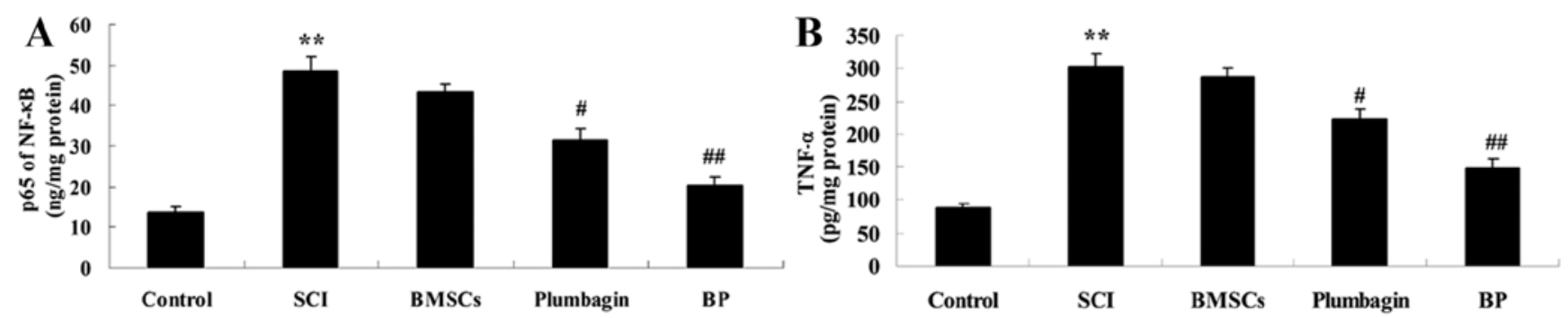

Figure 6. Treatment with bone marrow mesenchymal stem cells (BMSCs) and plumbagin affect inflammation after spinal cord injury (SCI). Treatment with $\mathrm{BP}$ affected activities of (A) nuclear factor- $\mathrm{\kappa B}(\mathrm{NF}-\kappa \mathrm{B})$ p65 unit and (B) tumor necrosis factor- $\alpha$ (TNF- $\alpha$ ) after SCI. In the BP group, rats with SCI were treated with BMSCs ( $3 \times 10^{5}$ cells) administered by intraspinal injection using a Hamilton syringe and $20 \mathrm{mg} / \mathrm{kg}$ plumbagin for 5 consecutive days. The data are expressed as the means \pm SD and were analyzed using one-way ANOVA followed by Tukey's multiple comparison test. ${ }^{* *}$ p $<0.01$ compared with the control group; ${ }^{\#} \mathrm{p}<0.05$ compared with the SCI group; ${ }^{\# \#} \mathrm{p}<0.01$ compared with the SCI group. Control, control group; SCI, SCI group; BMSCs, BMSC-treated group; plumbagin, plumbagin-treated group; BP, BMSC + plumbagin-treated group.
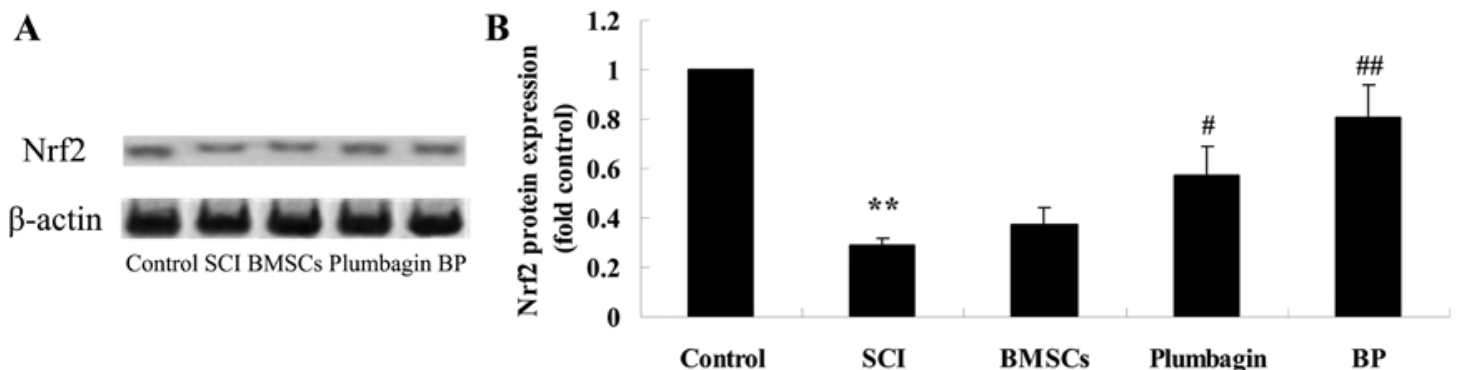

Figure 7. Treatment with bone marrow mesenchymal stem cells (BMSCs) and plumbagin affects the nuclear factor erythroid2-related factor 2 (Nrf2) pathway after spinal cord injury (SCI). Treatment with BP affects Nrf2 protein expression, as demonstrated by (A) western blot analysis and (B) the statistical analysis of the Nrf2 protein expression after SCI. In the BP group, rats with SCI were treated with BMSCs $\left(3 \times 10^{5}\right.$ cells) administered by intraspinal injection using a Hamilton syringe and $20 \mathrm{mg} / \mathrm{kg}$ plumbagin for 5 consecutive days. The data are expressed as the means $\pm \mathrm{SD}$ and were analyzed using one-way ANOVA followed by Tukey's multiple comparison test. ${ }^{* *} \mathrm{p}<0.01$ compared with the control group; ${ }^{*} \mathrm{p}<0.05$ compared with the SCI group; ${ }^{\# \#} \mathrm{p}<0.01$ compared with the SCI group. Control, control group; SCI, SCI group; BMSCs, BMSC-treated group; plumbagin, plumbagin-treated group; BP, BMSC + plumbagin-treated group.
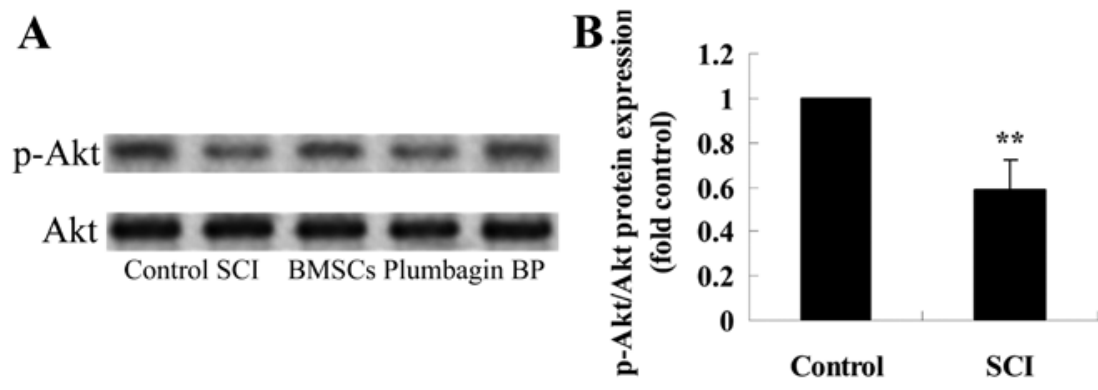

Figure 8. Treatment with bone marrow mesenchymal stem cells (BMSCs) and plumbagin affects phosphoinositide 3-kinase (PI3K)/Akt pathways after spinal cord injury (SCI). Treatment with BP affected p-Akt protein expression, as shown by (A) western blot analysis and (B) statistical analysis of p-Akt protein expression after SCI. In the BP group, rats with SCI were treated with BMSCs $\left(3 \times 10^{5}\right.$ cells) administered by intraspinal injection using a Hamilton syringe and $20 \mathrm{mg} / \mathrm{kg}$ plumbagin for 5 consecutive days. The data are expressed as the means \pm SD and were analyzed using one-way ANOVA followed by Tukey's multiple comparison test. ${ }^{* *} \mathrm{p}<0.01$ compared with the control group; ${ }^{*} \mathrm{p}<0.05$ compared with the SCI group. Control, control group; SCI, SCI group; BMSCs, BMSC-treated group; plumbagin, plumbagin-treated group; BP, BMSC + plumbagin-treated group.

significant difference between the expression of $\mathrm{p}$-Akt/Akt in the BP-treated group and the BMSC-treated group (Fig. 8).

Treatment with BP affects the expression of p-p38 MAPK after $S C I$. To further examine the possible mechanism of treatment with BP on SCI, p-p38 MAPK protein expression was evaluated using western blot analysis. We found that in the SCI group p-p38 MAPK protein expression was significantly increased when compared to the control group (Fig. 9). BMSCs inhibited the expression in a non-statistically significant manner. Plumbagin significantly inhibited the protein expression of p-p38 MAPK when compared to the SCI group. Moreover, treatment with BP significantly inhibited the protein expression of p-p38 MAPK compared with the SCI group (Fig. 9).

Treatment with BP affects the ERK pathways after SCI. To further survey the possible effect of treatment with BP on SCI, p-ERK and ERK protein expression were estimated using western blot analysis. In the SCI group, p-ERK/ERK expression was significantly inhibited when compared to the control group (Fig. 10). We found no significant inter-group difference between the SCI group and the BMSC-treated group. The results 
A

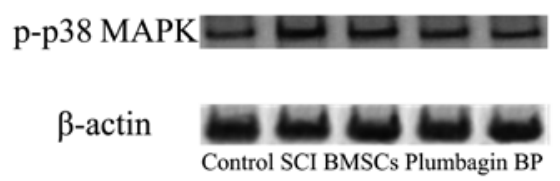

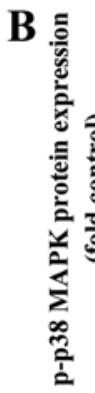

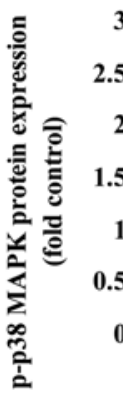

3 5

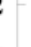

1 -

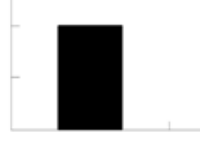

Control
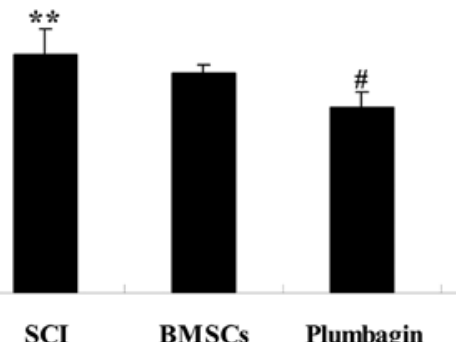

Plumbagin

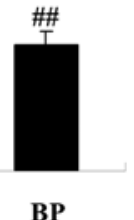

BP

Figure 9. Treatment with bone marrow mesenchymal stem cells (BMSCs) and plumbagin affects expression of p-p38 mitogen-activated protein kinase (MAPK) after spinal cord injury (SCI). Treatment with BP affected p-p38 MAPK protein expression, as measured using (A) western blot analysis and (B) statistical analysis of the p-p38 MAPK protein expression after SCI. In the BP group, rats with SCI were treated with BMSCs (3x10 5 cells) administered by intraspinal injection using a Hamilton syringe and $20 \mathrm{mg} / \mathrm{kg}$ plumbagin for 5 consecutive days. The data are expressed as the means \pm SD and were analyzed using one-way ANOVA followed by Tukey's multiple comparison test. ${ }^{* *}$ p $<0.01$ compared with the control group; ${ }^{\#}<0.05$ compared with the SCI group; ${ }^{\#} \mathrm{p}<0.01$ compared with the SCI group. Control, control group; SCI, SCI group; BMSCs, BMSC-treated group; plumbagin, plumbagin-treated group; BP, BMSC + plumbagin-treated group.

A

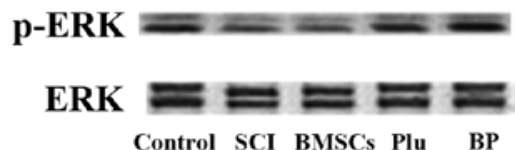

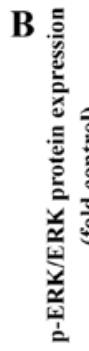

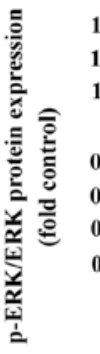

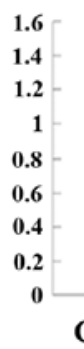
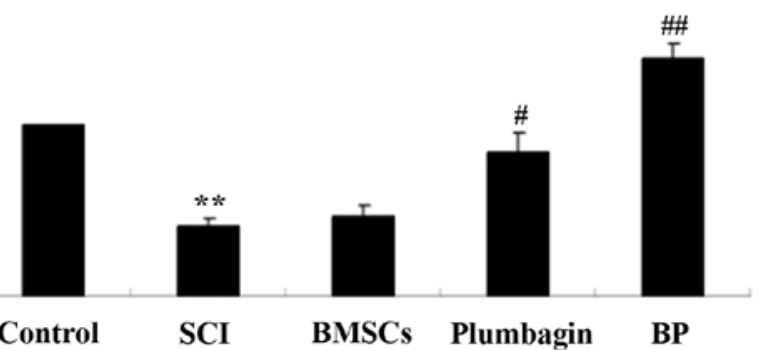

Figure 10. Treatment with bone marrow mesenchymal stem cells (BMSCs) and plumbagin affects extracellular-signal-regulated kinase (ERK) pathways after spinal cord injury (SCI). Treatment with BP affected p-ERK protein expression, as measured using (A) western blot analysis and (B) statistical analysis of the p-ERK protein expression after SCI. In the BP group, rats with SCI were treated with BMSCs ( $3 \times 10^{5}$ cells) administered by intraspinal injection using a Hamilton syringe and $20 \mathrm{mg} / \mathrm{kg}$ plumbagin for 5 consecutive days. The data are expressed as the means \pm SD and were analyzed using one-way ANOVA followed by Tukey's multiple comparison test. ${ }^{* *}$ p $<0.01$ compared with the control group; ${ }^{\#} \mathrm{p}<0.05$ compared with the SCI group; ${ }^{\# \#}$ p $<0.01$ compared with the SCI group. Control, control group; SCI, SCI group; BMSCs, BMSC-treated group; plumbagin (Plu), plumbagin-treated group; BP, BMSC + plumbagin-treated group.

revealed that plumbagin treatment significantly increased the p-ERK/ERK rate when compared to the SCI group. The p-ERK/ERK rate of expression in rats treated with BP was considerably higher than in the SCI-treated group (Fig. 10).

\section{Discussion}

With the increasing economic development of society, the incidence of SCI increases year by year, and it seriously affects the quality of life of patients, and has serious adverse effects on the patient's family and the community (15). The disintegration and necrosis of a large number of neuronal cells in tissues of patients after SCI is one of the major causes of the reduction in limb movement and other sensory dysfunctions in the area below the injury (16). At present, there is no effective method for paraplegics with SCI to gain restored function (17). Thus, promoting the regeneration of nerve cells and recovering the structure and the function of spinal cord are important and difficult issues which face neuroscientists (18). In the present study, treatment with BP was noted to improve locomotor recovery and reduce spinal cord water content and MPO activity in rats with SCI. Mansilla et al have previously reported that human mesenchymal stem cells alleviated spinal cord injuries (19). Wang et al have demonstrated that transplanted BMSCs reduce MPO activity in rats with pancreatitis-associated lung injury (20). Zhang et al showed that plumbagin protects against oxidative stress and inflammation in rats with SCI (12). Thus, we suggest that treatment with $\mathrm{BP}$ is a promising strategy for treating SCI.

The SCI mechanism has not yet been fully elucidated, but the present study indicates that oxidative stress plays an important role in it. Currently, it is thought that many oxygen free radicals (OFRs) are generated in SCI pathogenesis, which have a trigger-like effect on damage to the body, and calcium overload is a final common result of cellular damage (21). SOD is an enzyme that catalyzes the disproportionation of superoxide anion, and is a major antioxidative enzyme and free-radical scavenger in cells; it protects cells against oxygen free radicals, and the level of SOD indicates the level of protective effectiveness of cells from toxic damage caused by free radicals (22). MDA is the end product of lipid peroxidation, and MDA levels directly reflect the level of free radicals, of which the content is an important indicator of tissue damage (23). The determination of SOD activity and MDA content indirectly reflect the ability of the body's antioxidant system.

Inflammation plays an important role in the pathogenesis of SCI, and the inflammatory response of local damaged tissue increases the degree of secondary SCI (24). The secondary inflammatory environment caused by local damage leads to cellular necrosis and apoptosis at the SCI site, and irreversible pathological changes occur, thereby increasing SCI (25). Previous research has reported that neutrophils aggregate to the site of injury to release elastase and other substances, 
increasing tissue edema, necrosis and promoting neuronal and oligodendrocyte apoptosis, thus resulting in the emergence of localized glial scars (26). The results from our study showed that treatment with BP suppressed oxidative stress and inflammation following SCI. Tu et al suggested that bone marrow-derived mesenchymal stem cells attenuate oxidative stress and systemic inflammation in rats with severe acute pancreatitis (27). Checker et al demonstrated that plumbagin abrogates lipopolysaccharide-induced oxidative stress, inflammatory oxidative stress, inflammation and endotoxic shock (28). In the context of these results, we found in the present study that the anti-oxidative and anti-inflammatory effects of BP exert a certain protective effect against damage caused by SCI.

In the body, the Nrf2/ARE signaling pathway acts as an important endogenous anti-oxidative stress pathway, which encodes and modulates the expression of various antioxidant genes, enhances cellular resistance to oxidative stress, and thereby reduces the damage to the body caused by oxidative stress (29). When the spinal cord suffers from oxidative stress injury, the intracellular antioxidant defense system is essential for reducing the neuronal damage caused, and the Nrf2/ARE signaling pathway has been noted as the most important endogenous anti-oxidative stress pathway. Thus far, it has been noted that more than 200 endogenous genes can be encoded, and are regulated by the Nrf2/ARE signaling pathway. As the major regulatory protein of the Nrf2/ARE signaling pathway, antioxidant enzymes do not only catalyze free radicals into non-toxic substances, but also strengthen their water solubility to exclude them, which is an important factor in maintaining the redox balance of the body (30). In the present study, we found that BP therapy increased the suppressed $\mathrm{Nrf} 2$ expression in rats with SCI. Son et al have demonstrated the neuroprotective effects of plumbagin, and also demonstrated that it protects against cerebral ischemia through activation of Nrf2/ARE (31). Cho et al demonstrated that mesenchymal stem cells promote the CCl4-inhibited Nrf2 expression in rats with liver injury (32). In addition, we suggest that the protective effect of BP on SCI damage is involved in the activation of the Nrf2 signaling pathway.

Akt is an important factor in biological signaling pathways, and constitutes an important part of the PI3K/Akt signaling pathway, which inhibits apoptosis, promotes cell survival and plays an important role in the angiogenesis process (33). The PI3K/Akt pathway is involved in the growth and differentiation of neurons, inhibiting neuronal apoptosis and promoting axonal grow th and mediating synaptic plasticity and many other cellular processes, and it plays an important role in nerve physiological and pathological processes (34). It has been previously demonstrated that after optic nerve and hypoglossal nerve injury in rodents, increased Akt expression causes anti-neuronal apoptosis. After traumatic brain injury and ischemic brain injury in rats, Akt activity in neurons was significantly increased, and neuronal apoptosis was suppressed (35). In the present study, we discovered that administration of BP significantly increased the level of p-Akt/Akt compared to the SCI group. Chen et al have reported that BMSCs upregulated p-ERK and p-Akt through multiple pathways (36). Checker et al showed that plumbagin inhibits inflammatory responses but does not affect the phosphorylation of Akt (37). Moreover, we suggest that the protective effect which BP exerts against SCI damage involves the p-Akt signaling pathway.
MAPK is a type of serine/threonine protein kinase in the cell, which exists in the majority of mammals in the cytoplasm and nuclei, and can be stimulated for extracellular signaling to the cells and their nuclei, and thus lead to cell biological reactions, the regulation of cell proliferation, as well as differentiation, development and apoptosis (38). It is known that p-p38 MAPK is involved in nerve cell apoptosis and in the process of signal transduction. It has also been noted that p-p38 MAPK is involved in the acute inflammation caused by the SCI-induced apoptosis of neurons and glial cells (39). In the present study, treatment with BP significantly inhibited the protein expression of p-p38 MAPK in rats with SCI. Wang et al have suggested that the anti-inflammatory effect of plumbagin mediates the inhibitory effect of MAPK signaling on inflammation (40). Wang et al have reported that BMSCs promote cell proliferation, but do not influence p38 MAPK in rats (41). The reason for these various conclusions is likely the dosage or disinfecting times of BMSCs, and discussion in future studies is thus necessary.

Previous research has demonstrated that ERK1/2 exists in the central nervous system, and it participates in a variety of physiological and pathological processes (42). Research has shown that SCI, excitotoxicity and transection injury leads to elevated levels of p-ERK1/2, and in cases of contusion and excitotoxicity, ERK1/2 phosphorylation is increased significantly in the areas adjacent to the damage (43). In the present study, treatment with BP significantly increased p-ERK1/2 in SCI rats. Zhang et al demonstrated that BMSCs promoted osteoblast differentiation through upregulation of the ERK1/2 signaling pathway (44). Yang et al suggested that plumbagin activates ERK1/2 in 3T3-L1 cells (45). Thus, we suggest that the activation of ERK1/2 increases the protective effect which BP exerts againsts SCI damage.

In conclusion, in the present study, we identified that treatment with BP alleviates SCI through its effects on oxidative stress, inflammation, anti-apoptotis, the activation of $\mathrm{Nrf2}$, suppression of PI3K/Akt and p-p38 MAPK, and the upregulation of $\mathrm{p}$-ERK1/2.

\section{References}

1. Jiang SH, Tu WZ, Zou EM, Hu J, Wang S, Li JR, Wang WS, He R, Cheng RD and Liao WJ: Neuroprotective effects of different modalities of acupuncture on traumatic spinal cord injury in rats. Evid Based Complement Alternat Med 2014: 431580, 2014.

2. Nardone R, Höller Y, Brigo F, Orioli A, Tezzon F, Schwenker K, Christova M, Golaszewski S and Trinka E: Descending motor pathways and cortical physiology after spinal cord injury assessed by transcranial magnetic stimulation: a systematic review. Brain Res:139-154, 2014

3. Chen X, He F, Zhong DY and Luo ZP: Acoustic-frequency vibratory stimulation regulates the balance between osteogenesis and adipogenesis of human bone marrow-derived mesenchymal stem cells. BioMed Res Int 2015: 540731, 2015.

4. Lin H, Luo X, Jin B, Shi H and Gong H: The effect of EPO gene overexpression on proliferation and migration of mouse bone marrow-derived mesenchymal stem cells. Cell Biochem Biophys: Nov 14, 2014 (Epub ahead of print).

5. Munoz JL, Greco SJ, Patel SA, Sherman LS, Bhatt S, Bhatt RS, Shrensel JA, Guan YZ, Xie G, Ye JH, et al: Feline bone marrowderived mesenchymal stromal cells (MSCs) show similar phenotype and functions with regards to neuronal differentiation as human MSCs. Differentiation 84: 214-222, 2012.

6. Song M, Jue SS, Cho YA and Kim EC: Comparison of the effects of human dental pulp stem cells and human bone marrow-derived mesenchymal stem cells on ischemic human astrocytes in vitro. J Neurosci Res 93: 973-983, 2015. 
7. Tian L, Yin D, Ren Y, Gong C, Chen A and Guo FJ: Plumbagin induces apoptosis via the $\mathrm{p} 53$ pathway and generation of reactive oxygen species in human osteosarcoma cells. Mol Med Rep 5: 126-132, 2012

8. Luo P, Wong YF, Ge L, Zhang ZF, Liu Y, Liu L and Zhou H: Anti-inflammatory and analgesic effect of plumbagin through inhibition of nuclear factor- $\kappa \mathrm{B}$ activation. J Pharmacol Exp Ther 335: 735-742, 2010.

9. Subramaniya BR, Srinivasan G, Sadullah SS, Davis N, Subhadara LB, Halagowder D and Sivasitambaram ND: Apoptosis inducing effect of plumbagin on colonic cancer cells depends on expression of COX-2. PLoS One 6: e18695, 2011.

10. Ersahın M, Toklu HZ, Erzık C, Akakin D, Tetık S, Sener G and Yeğen BC: Ghrelin alleviates spinal cord injury in rats via its anti-inflammatory effects. Turk Neurosurg 21: 599-605, 2011.

11. Li F, Fei D, Sun L, Zhang S, Yuan Y, Zhang L, Zhao K, Li R and $\mathrm{Yu} \mathrm{Y}$ : Neuroprotective effect of bone marrow stromal cell combination with atorvastatin in rat model of spinal cord injury. Int J Clin Exp Med 7: 4967-4974, 2014

12. Zhang W, Cheng L, Hou Y, Si M, Zhao YP and Nie L: Plumbagin protects against spinal cord injury-induced oxidative stress and inflammation in Wistar rats through Nrf-2 upregulation. Drug Res (Stuttg) 65: 495-499, 2014

13. Chio CC, Lin JW, Chang MW, Wang CC, Kuo JR, Yang CZ and Chang CP: Therapeutic evaluation of etanercept in a model of traumatic brain injury. J Neurochem 115: 921-929, 2010.

14. Vink R, Young A, Bennett CJ, Hu X, Connor CO, Cernak I and Nimmo AJ: Neuropeptide release influences brain edema formation after diffuse traumatic brain injury. Acta Neurochir Suppl 86: 257-260, 2003.

15. Wang T, Yuan W, Liu Y, Zhang Y, Wang Z, Zhou X, Ning G, Zhang L, Yao L, Feng S and Kong X: The role of the JAK-STAT pathway in neural stem cells, neural progenitor cells and reactive astrocytes after spinal cord injury. Biomed Rep 3: 141-146, 2015.

16. Chen MH, Ren QX, Yang WF, Chen XL, Lu C and Sun J: Influences of HIF-l $\alpha$ on Bax/Bcl-2 and VEGF expressions in rats with spinal cord injury. Int J Clin Exp Pathol 6: 2312-2322, 2013

17. Bransford RJ, Chapman JR, Skelly AC, Vanalstyne EM: What do we currently know about thoracic spinal cord recovery and outcomes? A systematic review. J Neurosurg Spine 17 (Suppl 1): 52-64, 2012.

18. Adams M and Cavanagh JF: International Campaign for Cures of Spinal Cord Injury Paralysis (ICCP): another step forward for spinal cord injury research. Spinal Cord 42: 273-280, 2004

19. Mansilla E, Marin GH, Sturla F, Drago HE, Gil MA, Salas E, Gardiner MC, Piccinelli G, Bossi S, Salas E, et al: Human mesenchymal stem cells are tolerized by mice and improve skin and spinal cord injuries. Transplant Proc 37: 292-294, 2005.

20. Wang L, Tu XH, Zhao P, Song JX and Zou ZD: Protective effect of transplanted bone marrow-derived mesenchymal stem cells on pancreatitis-associated lung injury in rats. Mol Med Rep 6: 287-292, 2012

21. Qayumi AK, Janusz MT, Jamieson WR and Lyster DM: Pharmacologic interventions for prevention of spinal cord injury caused by aortic crossclamping. J Thorac Cardiovasc Surg 104 256-261, 1992

22. Cuevas P, Reimers D, Carceller F and Jimenez A: Ischemic reperfusion injury in rabbit spinal cord: protective effect of superoxide dismutase on neurological recovery and spinal infarction. Acta Anat (Basel) 137: 303-310, 1990

23. Koc RK, Akdemir H, Karakücük EI, Oktem IS and Menkü A Effect of methylprednisolone, tirilazad mesylate and vitamin $\mathrm{E}$ on lipid peroxidation after experimental spinal cord injury. Spinal Cord 37: 29-32, 1999.

24. Zhang X, Chen C, Ma S, Wang Y, Zhang X and Su X: Inhibition of monocyte chemoattractant peptide-1 decreases secondary spinal cord injury. Mol Med Rep 11: 4262-4266, 2015

25. Yao AH, Jia LY, Zhang YK, Ma QR, Cheng P, Liu L, Ju G and Kuang F: Early blockade of TLRs MyD88-dependent pathway may reduce secondary spinal cord injury in the rats. Evid Based Complement Alternat Med 2012: 591298, 2012.

26. Kadota R, Koda M, Kawabe J, Hashimoto M, Nishio Y, Mannoji C, Miyashita T, Furuya T, Okawa A, Takahashi K and Yamazaki M: Granulocyte colony-stimulating factor (G-CSF) protects oligodendrocyte and promotes hindlimb functional recovery after spinal cord injury in rats. PLoS One 7: e50391, 2012.

27. Tu XH, Song JX, Xue XJ, Guo XW, Ma YX, Chen ZY, Zou ZD and Wang L: Role of bone marrow-derived mesenchymal stem cells in a rat model of severe acute pancreatitis. World J Gastroenterol 18: 2270-2279, 2012.
28. Checker R, Patwardhan RS, Sharma D, Menon J, Thoh M, Sandur SK, Sainis KB and Poduval TB: Plumbagin, a vitamin K3 analogue, abrogates lipopolysaccharide-induced oxidative stress, inflammation and endotoxic shock via NF- $\mathrm{BB}$ suppression. Inflammation 37: 542-554, 2014.

29. Benedict AL, Mountney A, Hurtado A, Bryan KE, Schnaar RL, Dinkova-Kostova AT and Talalay P: Neuroprotective effects of sulforaphane after contusive spinal cord injury. J Neurotrauma 29: 2576-2586, 2012

30. Wang X, de Rivero Vaccari JP, Wang H, Diaz P, German R, Marcillo AE and Keane RW: Activation of the nuclear factor E2-related factor 2/antioxidant response element pathway is neuroprotective after spinal cord injury. J Neurotrauma 29: 936-945, 2012.

31. Son TG, Camandola S, Arumugam TV, Cutler RG, Telljohann RS, Mughal MR, Moore TA, Luo W, Yu QS, Johnson DA, et al: Plumbagin, a novel Nrf2/ARE activator, protects against cerebral ischemia. J Neurochem 112: 1316-1326, 2010.

32. Cho KA, Woo SY, Seoh JY, Han HS and Ryu KH: Mesenchymal stem cells restore CCl4-induced liver injury by an antioxidative process. Cell Biol Int 36: 1267-1274, 2012.

33. Liu W, Zhang L, Shi J, Liu Y, Zhou L, Hou K, Qu X and Teng Y: Clinical significance of pAkt and pErk1/2 expression in earlystage breast cancer patients treated with anthracycline-based adjuvant chemotherapy. Oncol Lett 9: 1707-1714, 2015.

34. Ha KS, Kim KM, Kwon YG, Bai SK, Nam WD, Yoo YM, Kim PK, Chung HT, Billiar TR and Kim YM: Nitric oxide prevents 6-hydroxydopamine-induced apoptosis in PC12 cells through cGMP-dependent PI3 kinase/Akt activation. FASEB J 17: 1036-1047, 2003.

35. Chuenkova MV and Pereiraperrin M: Neurodegeneration and neuroregeneration in Chagas disease. Adv Parasitol 76: 195-233, 2011.

36. Chen TL, Zhu GL, Wang JA, Wang Y, He XL and Jiang J: Apoptosis of bone marrow mesenchymal stem cells caused by hypoxia/reoxygenation via multiple pathways. Int J Clin Exp Med 7: 4686-4697, 2014.

37. Checker R, Sharma D, Sandur SK, Subrahmanyam G, Krishnan S, Poduval TB and Sainis KB: Plumbagin inhibits proliferative and inflammatory responses of T cells independent of ROS generation but by modulating intracellular thiols. J Cell Biochem 110: 1082-1093, 2010.

38. Shin BA, Yoo HG, Kim HS, Kim MH, Hwang YS, Chay KO, Lee KY, Ahn BW and Jung YD: p38 MAPK pathway is involved in the urokinase plasminogen activator expression in human gastric SNU-638 cells. Oncol Rep 10: 1467-1471, 2003.

39. Galan-Arriero I, Avila-Martin G, Ferrer-Donato A, Gomez-Soriano J, Bravo-Esteban E and Taylor J: Oral administration of the p38 $\alpha$ MAPK inhibitor, UR13870, inhibits affective pain behavior after spinal cord injury. Pain 155: 2188-2198, 2014

40. Wang T, Wu F, Jin Z, Zhai Z, Wang Y, Tu B, Yan W and Tang T: Plumbagin inhibits LPS-induced inflammation through the inactivation of the nuclear factor-kappa B and mitogen activated protein kinase signaling pathways in RAW 264.7 cells. Food Chem Toxicol 64: 177-183, 2014

41. Wang Y, Wang J, Bai D, Song J, Ye R, Zhao Z, Lei L, Hao J, Jiang C, Fang S, et al: Cell proliferation is promoted by compressive stress during early stage of chondrogenic differentiation of rat BMSCs. J Cell Physiol 228: 1935-1942, 2013.

42. Pernet V, Hauswirth WW and Di Polo A: Extracellular signalregulated kinase 1/2 mediates survival, but not axon regeneration, of adult injured central nervous system neurons in vivo. J Neurochem 93: 72-83, 2005.

43. Shi B, Ding J, Liu Y, Zhuang X, Zhuang X, Chen X and Fu C: ERK1/2 pathway-mediated differentiation of IGF-1-transfected spinal cord-derived neural stem cells into oligodendrocytes. PLoS One 9: e106038, 2014.

44. Zhang P, Wu Y, Jiang Z, Jiang L and Fang B: Osteogenic response of mesenchymal stem cells to continuous mechanical strain is dependent on ERK1/2-Runx2 signaling. Int J Mol Med 29: 1083-1089, 2012

45. Yang SJ, Chang SC, Wen HC, Chen CY, Liao JF and Chang CH: Plumbagin activates ERK1/2 and Akt via superoxide, Src and PI3-kinase in 3T3-L1 cells. Eur J Pharmacol 638: 21-28, 2010. 